\title{
Frequency and Amplitude Measurement of A Cantilever Beam Using Image Processing
}

\author{
With A Feedback System
}

\author{
Sohaib Z Khan* \\ Department of Mechanical Engineering, Faculty of \\ Engineering, Islamic University Madina, \\ Madina, Saudi Arabia \\ *email: szkhan@iu.edu.sa ; \\ Consultation.DrSohaib@gmail.com \\ Muhammad A Khan \\ School of Aerospace, Transport and Manufacturing, \\ Cranfield, Cranfield University, \\ United Kingdom
}

\author{
Umair Ahmed, \\ Sallar Qazi, Salman Nisar \\ Department of Engineering Sciences, PNEC, \\ National University of Sciences and Technology, \\ Karachi, Pakistan
}

Kamran A Khan

Department of Aerospace Engineering, Khalifa University of Science, Technology and Research (KUSTAR),

Abu Dhabi, UAE

\author{
Shafiq-ur-Rehman Qureshi \\ Department of Engineering Sciences, PNEC, \\ National University of Sciences and Technology, \\ Karachi Pakistan
}

\section{INTRODUCTION}

\begin{abstract}
Image processing techniques can be utilized in analyzing amplitude and frequency of vibrating structures. It is a form of non-contact method which is suitable for cases where application of contact devices could alter the frequency of structure. This paper covers the study based on vision system that performs amplitude and frequency measurement of a cantilever beam in near real time, using image processing and computer vision toolbox in MATLAB. The vision system then detects changes in amplitude followed by feedback mechanism to ensure operation at resonance frequency. The system includes a high speed camera which is able to detect amplitude and frequency of cantilever beam vibrating at a frequency with the help of mechanical exciter. The high speed camera captures images of the beam, that are processed by a MATLAB script for evaluation of amplitude and frequency. To locate amplitude of the vibrating beam, centroid recognition technique is used which tracks the centroids of the beam in consecutive frames and plots number of pixels moved by the centroid with respect to time. Later, frequency is found out on the basis of intensity change over the time. Amplitude analysis is done at different frequencies which are automatically adjusted with the help of microcontroller to determine the resonance point. Exciter continues to vibrate at the resonant frequency until a change in amplitude is detected, implying the formation of crack. At which point the system adjusts its vibrating frequency accordingly to adjust with the new resonant frequency. This paper covers proper experimental procedure backed with the results.
\end{abstract}

Keywords-Image Processing, cantilever beam, resonance, high speed camera
In recent years, the research on modeling and studying the characteristics of vibrating structures using image-based techniques have considerably increased. This is because, other devices for vibration measurement are contact based and can be error prone for the bodies with a significantly smaller mass and where precise vibration measurement is necessary.

One researcher determined the frequency response of a cantilever beam by analyzing the image sequence of a target attached to the vibrating beam at the sub-pixel level. That target tracking algorithm computes central co-ordinates of the target from a sequence of images and gives a displacement time relationship that is transformed into frequency domain using Discrete Fourier Transform (DFT). With sub-pixel accuracy, the target extraction algorithm can cope with smaller changes in position which enables detection of vibrations of a higher frequency and increases efficiency [1].

Another technique an author used is selecting a cantilever beam and dividing it into seven regions of interest while exciting the beam from one end with the Exciter, and recording a sequence of images. The recorded images are then subject to preliminary image processing to improve the visibility of concerned points. Seven regions of interest are then created in the regions of points and center of gravity of each point of every picture is measured in MATLAB. Two successive images are then compared and the displacement that has occurred between the center of gravity of individual points is measured. Displacement-time graph is plotted for all displacement points to obtain the frequency at which it is vibrating [2]. 
Using the technique of Image-based tracking Laser Doppler Vibrometer (iTLDV), P. Castellini [3] developed a system for tracking of arbitrary motions; the system is feedback controlled and allows to measure vibrations of moving target by driving the moving mirrors via position signals of the target obtained from a CCD camera and an image processing algorithm. The tracking system developed has been applied to an industrial test case subject to self-excited vibrations, to verify performances and limits in operating conditions.

In one of the papers, Belen Ferrer, presented another method of tracking the frequency of structures by recording the video with a high FPS camera and then after, tracking the changes in the two successive frames and quantifying them. Fast Fourier Transform is then performed and frequency of the object is calculated [4].

Considering FFT as a basis for frequency analysis of acoustic signals Tomáš Hararika, Jozef Bockoa and Kristína (2012) examined the mechanical frequency of cantilever beam. The methodology used was to record the noise generated by vibrating system into a digital file using microphone and then analyzed the data using Fast Fourier Transformation in MATLAB [5].

In a paper by Xiaoteng, an image acquisition and processing system are developed to observed floc size distribution. The vision system is utilized to capture images which are then processed by MATLAB image processing toolbox for output. A micro-controller is also used for the proposed system which serves as a feedback by controlling light source and frame trigger of camera. The processed floc size and their statistical distributions are confirmed with two available sample sets [6].

Using motion magnification for calculating displacements Justin [7] estimated modal shapes of a cantilever beam. The beam motion was simultaneously measured by a laser vibrometer, an accelerometer and a high fps camera. The comparison between the data derived from all devices showed that the camera extracted mode shapes quite well compared with others devices. This verification measurement gives another reasonable confidence that frequency of a beam can successfully be measured by using image processing techniques and also proved that camera can be a much better option for instrumenting a structure as compared with other instruments.

In a paper by N. Ab Azara [8], a vision system was proposed which was able to detect and count overlapping geometrical object with high accuracy and efficiency. Formulated script in MATLAB was based on pattern recognition, feature extraction and image pixel value comparison technique.

In a research paper by Xiaolei Zhua, a modelling based approach is proposed to develop a finite element model of aluminum foam. Initially, MATLAB image processing is used to deal with synchrotron X-ray computed tomography scanning images of real aluminum foam and later forming a geometric model based on image processing results [9]. Moreover, twostep mesh method is employed to mesh the geometrical model by appropriate selection of the node, and then establish the FEM of aluminum foam.

\section{METHODOLOGY}

The main aim of this work is to develop an autonomous system that complies the vibrating cantilever beam to vibrate on its fundamental frequency even after the change in the fundamental frequency due to internal structural change during continuous oscillation. It has been shown in the literature that the during continuous vibration the natural frequency drops [10]. Studies have shown that the change in fundamental frequency is mainly due to the crack formation and crack propagation, which alters the integrity of the internal structure of the material.

For vibration analysis of small and lightweight structures contact sensors add mass which can alter the fundamental frequency of the structure. Therefore, in this work, non-contact image processing technique is suggested, which not only detect the fundamental frequency of vibrating cantilever beam but also modify the frequency of the shaker to keep the cantilever vibrating near to the maximum amplitude. The maximum amplitude (resonance) refers to near the fundamental frequency, once the frequency of the beam drops from the initial value.

\section{EXPERIMENTAL PROCEDURE}

\section{A. Physical Setup}

The fundamental modal frequency of the beam can be calculated using Timoshenko beam equation as shown in Eq. 1 [10]. In this equation, $E$ is the modulus of elasticity, $I_{e}$ is an equivalent second moment of inertia, $\rho$ is the density of material, $A$ is the cross-sectional area of thinner section, $l$ is the length of specimen and 1.875104 is a factor for end condition [10]. A non-prismatic shape of specimen is used in this work as shown in Figure 1 for accelerating testing. The edges allowed stress concentration around the radius to form cracks or in other words internal structural change of the material. This directly resulted in change in the fundamental frequency of the beam [10]. Except the length, the dimensions of the specimen remained the same. The change length was kept variable to have beams with different fundamental frequencies. In Eq. 2 which is used to calculate $I_{e}, t$ is constant thickness, while $B_{L}$ and $B_{R}$ are the breadths immediate to the left and right of the sudden change in cross-section, respectively. In this work the values of $B_{L}, B_{R}$ and $t$ are $20 \mathrm{~mm}, 10 \mathrm{~mm}$ and 0.5 $\mathrm{mm}$, respectively.

$$
\begin{aligned}
& f_{n}=0.5596 \sqrt{\frac{E I_{e}}{\rho A l^{4}}} \\
& I_{e}=\left(\frac{B_{L} \times B_{R} \times t^{3}}{6\left(B_{L}+B_{R}\right)}\right)
\end{aligned}
$$

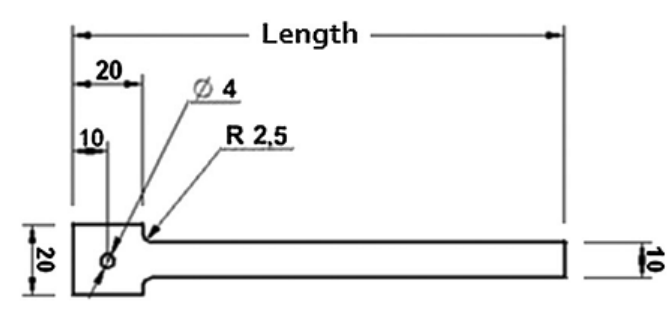

Fig. 1: Geometry of the cantilever beam specimen 
The experimental setup can be distributed as a vibrating mechanism, data acquisition and data processing. The vibrating mechanism comprises a model exciter (Tenlee Piezotronics Inc Model MS-100), amplifier (Veredan Technologies Model LA200) and a microcontroller Arduino DUE Atmel SAM3X8E ARM Cortex-M3 CPU.

Modal exciter was used for excitation of a cantilever beam with the ability to provide inputs large enough to result in easily measured responses and can be used to impart a variety of both sinusoidal and non-sinusoidal input signals to the structure. To operate exciter mechanism and to provide the necessary energy, a power amplifier was used which was operated with adjustable gain. The exciter was connected to the computer via microcontroller to provide feedback from the computer. Microcontroller, Arduino was used as a feedback controller as it is inexpensive, comparatively easy to program and compatible with MATLAB interface. Complete experimental setup is shown in Figure 2.

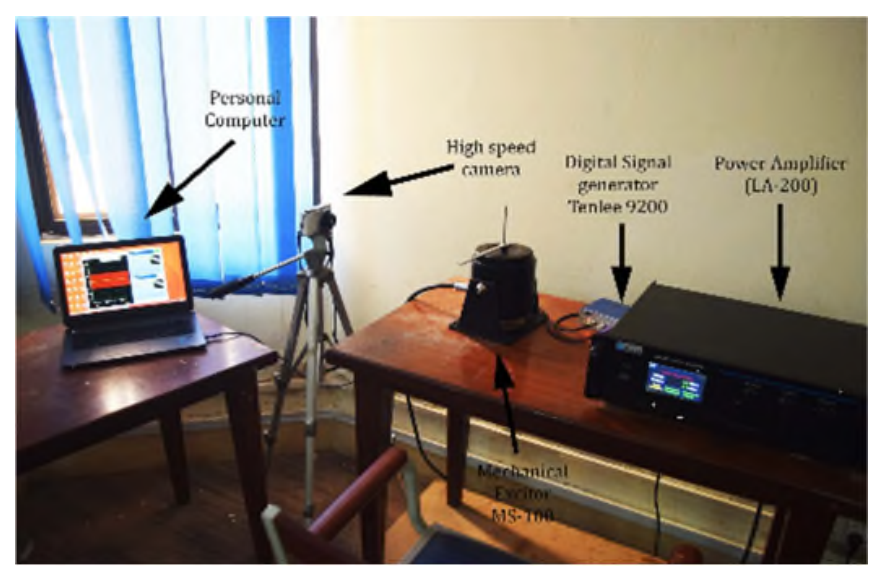

Fig. 2: Complete experimental setup with exciter and camera.

\section{B. Process}

The overall workflow of the experimental set is explained in Figure 3, At first, the exciter starts vibrating at a frequency arbitrarily set by the user for a specified number of frames. After acquiring the frames, the MATLAB code then runs the amplitude and frequency calculation scripts to calculate frequency and amplitude of the vibrating beam. The code then increases the vibration frequency and calculates amplitude and frequency of beam at this specified value. The two amplitude values are compared and if the second amplitude is higher than frequency of the system is further increased. This process is repeated until the point when the amplitude of the recorded value is less than previously recorded value. At that point, the code decreases the frequency to the average of the two frequencies and checks whether amplitude obtained at this point is higher than the highest recorded value or not. If the amplitude is higher than that frequency is determined to be the highest frequency. The exciter then keeps vibrating at this frequency and keeps checking for the drop in amplitude after every 300 frames or any number of frames the user deems suitable. As soon as the drop in amplitude is detected the system then displays a message. That detected drop in corresponding the frequency suggested that a crack has formed. The whole process then repeats to find the new resonant frequency of the cracked beam.

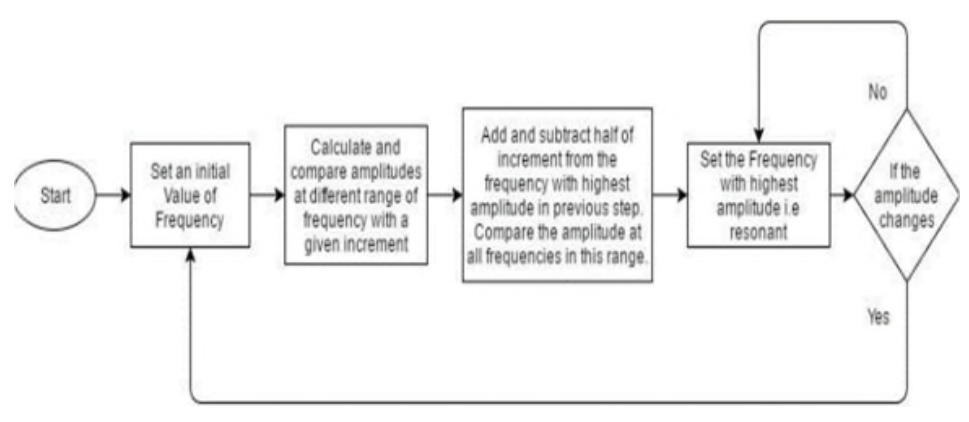

Fig 3: Decision making of micro-controller.

The algorithm is designed in such a manner that first microcontroller excites the specimen at frequencies with predetermined increments. Amplitude data obtained at different frequencies is calculated, compared and the frequency resulting in near highest amplitude is selected as the excitation frequency for specimen. In the next step, multiple iterations are done around that selected frequency to get a more accurate value of resonant frequency. The specimen will keep on vibrating at the resonant frequency until a change in amplitude is detected. If detected, the whole process will be repeated by the feedback system to find new resonance frequency.

For image acquisition two different cameras were used. Casio EXILIM EX - ZR1000 was used to capture images of the vibrating beam for frequencies up to $500 \mathrm{~Hz}$ with a maximum of 1000 frames per second (fps). Nikon DSLR was used for real-time lower frequencies up to $15 \mathrm{~Hz}$ at $30 \mathrm{fps}$.

MATLAB Image and Data Acquisition Toolboxes were used to interface between vibrating mechanism and image acquisition device to acquire videos. These toolboxes were easy to configure and access plug-in devices to alter acquisition properties as per the requirement.

\section{Image Processing}

Initially, the input parameters are defined in the MATLAB script which includes camera type, a region of interest, number of frames to be captured and serial port number. As defined by the parameters, the beam will start vibrating at a certain starting frequency and as defined by the user, 200 to 300 video frames will be captured to precisely trace the vibrating motion. After receiving a total number of specified video frames, the video input directly taken from the camera is recalled into MATLAB where it computes the amplitude and frequency of the vibrating beam.

The techniques employed for amplitude and frequency analysis of the video are the centroid determination method and pixel intensity detection at a point. For amplitude analysis, the centroid of the beam is calculated at each and every frame of the video, which is then plotted to give the amplitude graph of the beam as shown in Figure 4. 


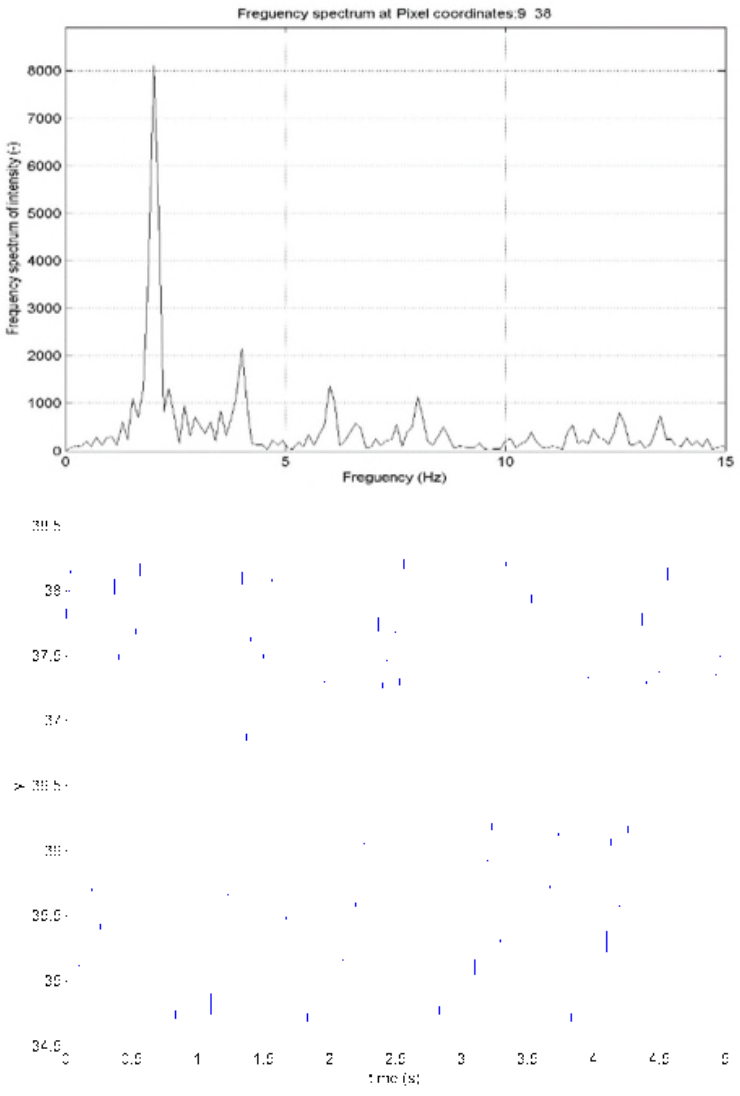

Fig 4: Frequency and amplitude at $2 \mathrm{~Hz}$.

\section{Frequency Analysis}

The digital image is a representation of a two-dimensional image as a finite set of digital values, called pixels containing digital numeric RGB color data. The basic concept used in this experiment for frequency analysis is that slight changes in intensity value of a monitored pixel caused by the vibration of structures can be captured by employing techniques such as Fast Fourier Transform (FFT). This technique is referred to as pixel intensity method.

Based on this approach, image data for processing and storage is transferred to MATLAB and a single pixel of the specimen is picked from the selected region of interest (ROI). This intensity point is determined by subtracting intensity values of corresponding pixels in two subsequent frames. The point that has most varying intensity is selected as the point of interest. The point selected is known to be the one which is continuously varying from frame to frame. This intensity point is shown in Figure 5.

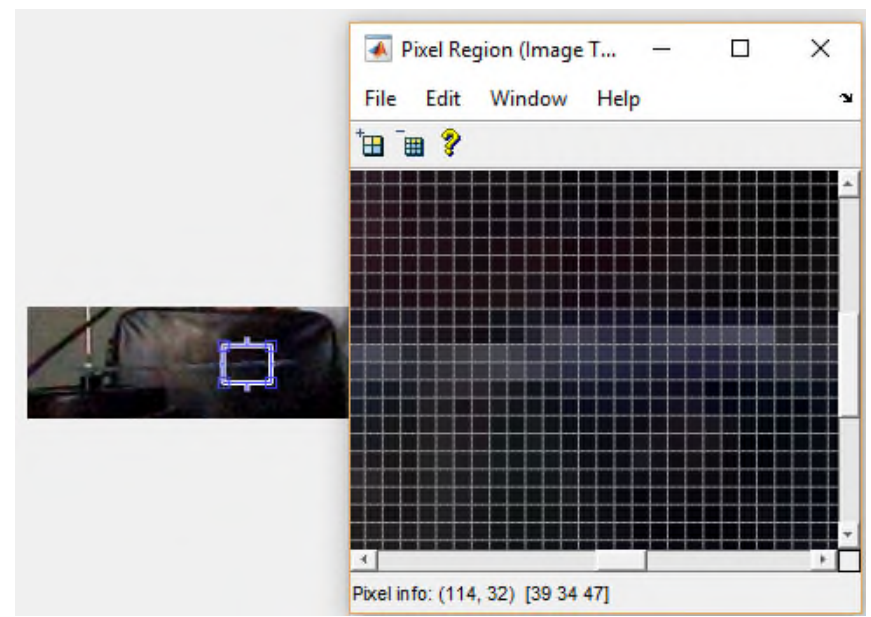

Fig. 5: Point of interest selection

Performing analysis on a small region of interest(ROI) results in a reduction in noise and time required for data acquisition. The selected point has pixel intensity in RGB values and its intensity is continuously varying from frame to frame. The pixel value of the selected point is then converted to the intensity value. Intensity is in actual, the greyscale equivalent of presented RGB colors and is obtained by using formula as shown in Eq 3, where, R, G, B (Red, Green and Blue, respectively) represent the specified intensity of the color in that pixel.

$$
I=0.2989 R+0.5870 G+0.1140 B
$$

This step of converting RGB value of the pixel to the grayscale value is repeated for that specified pixel throughout all the captured frames and the grayscale values are then merged into a matrix of the length equal to the number of frames of video.

Knowing the recording frame rate of the video, intensity values from each frame can be mapped versus time on the graph. Since, MATLAB acquires images at a set frame rate of 30 and plots it as one frame per second. Therefore, time scale needs to be calibrated to set it according to the real-time scale. This is achieved by dividing the total number of frames by 1000 and set the increment to be $10 \mathrm{e}-3$. The time-intensity diagram looks similar to and is proportional to a position-time diagram. If this is not the case, then the Fourier transform is likely to be inconclusive or unclear.

The changing variation of grayscale intensities over time is utilized and is converted into the frequency domain to obtain frequency. Once the time-intensity values are determined, a fast Fourier transform is applied to the signal. This transforms the data from the time domain into the frequency domain. Relatively tall spikes in these plots correlate to significant, measurable natural frequencies of the signal.

If there is too much noise in the signal, then this frequency analysis process is repeated with a different pixel, and is done until a perfect pixel is selected which could deliver correct results. There could also be additional peaks resulting from a poorly chosen color gradient or one that is too small. Further 
analysis with different pixel coordinates will help in determining when these effects occur.

The reason for using Pixel Intensity technique is because less information is needed to be provided for each pixel for performing FFT and the technique is both accurate as well as less time-consuming.

Calculating Pixel intensity change over time is less time consuming and in general, this analysis can be performed on any number of desired points and on any section of the beam whether symmetric or asymmetric. The following flowchart, as shown in Figure 6, explains the frequency analysis process.

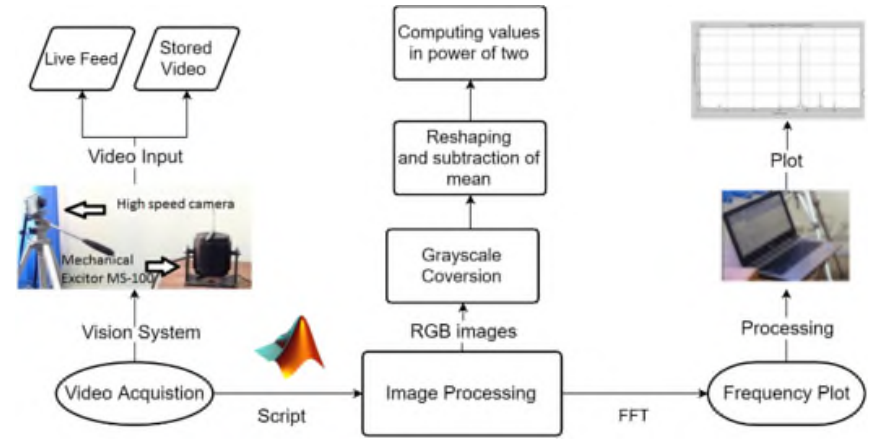

Fig. 6: Flowchart for the frequency analysis process

\section{E. Amplitude Analysis}

Maximum oscillation occurred when the external frequency applied to the beam matches that of its natural frequency. The amplitude analysis was carried out to determine the amplitude at different frequencies. The techniques range from very basic algorithm to more sophisticated probabilistic modeling techniques. To avoid both computational complexity and time, a relatively straightforward approach, centroid plotting is utilized and further to track centroid of the specimen for amplitude plotting the technique employed is known as Background Subtraction. In this technique, recorded video is first transferred to MATLAB using Image Acquisition Toolbox.

All the frames captured are then converted to grey scale to obtain uniform black and white images by setting an appropriate value of thresholding to differentiate between foreground and background. One reason for differentiating grey scale images from color images is that, for analysis less information is needed to be provided for each pixel and it helps detect changes more easily. This step converts the values to greyscale and smoothens the process for image dilation.

After Greyscale conversion, the frames become monotonous ranging from 0 to 255 on the intensity level. At this point, image dilation is performed using built-in MATLAB functions to reduce noise. Image dilation changes the nearest pixel values to that of the same color, and create a region which could be processed as one region.

In the next step, background is subtracted from all the frames leaving only the required beam area. This process clears the background and after performing thresholding, only the white and black region are left, as shown in Figure 7.

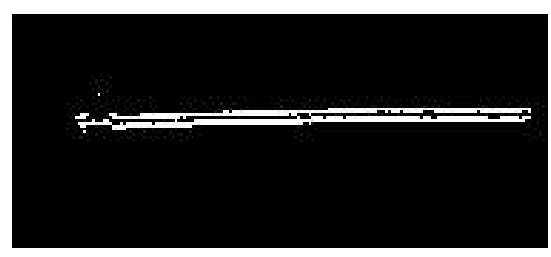

Fig. 7: Image of the beam after pre-processing.

As can be seen from the Figure 7, the cantilever beam now becomes a curved shape on a black screen; this happens for all the frames available in the Matrix stack. An area field is generated for this region and centroid of this area is then calculated in each image by defining it as a singular area. With the changing amplitude of the beam, the changing centroid values are stored in a separate variable for further analysis.

Amplitude-time plot is achieved by plotting the centroid values obtained with respect to the time; time is calibrated with the number of frames by dividing with the video frame rate. Commands for detecting maximum and minimum displacement points over the range of number of frames. These maximum and minimum will then be used during the feedback analysis to determine whether amplitude is increasing and decreasing.

Since the code is sensitive to even the slightest change in the immediate surrounding. Therefore, exceptional care needs to be taken while placing the camera. Only that region should be visible which is to be processed. This would increase process speed and accuracy. The flowchart shows in Figure 8, how the amplitude analysis code operates from the video acquisition step to the amplitude graph.

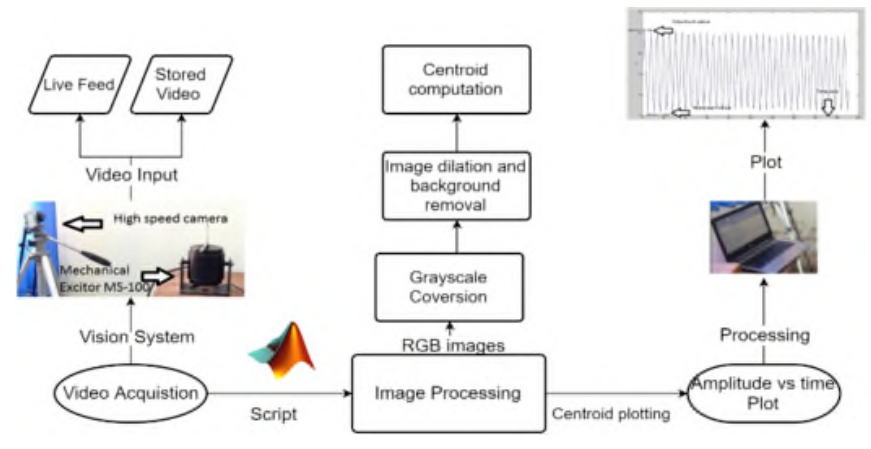

Fig. 8: Amplitude analysis process

\section{F. Feedback control mechanism}

After the development of frequency and amplitude analysis scripts, the focus shifts to design a feedback control mechanism for the achievement and adjustments towards the resonant frequency of the vibrating cantilever beam automatically without any user input.

The technique employed for the feedback control system is simple, it includes division of all the previous codes in the form of functions to reduce the redundancy in the code. In the beginning, the user must specify some input parameters, which are: 
- Camera acquisition device number

- Quality of the video

- Region of interest as defined in the live video feed part

- Camera capture framerate

- Number of frames to capture

- Computer port number being utilized

All these input parameters are then used in all the function to evaluate frequency and amplitude at different frequencies. The basic idea behind the working of the script is to use a microcontroller for providing the initial value of frequency to the exciter. Later the formulated script performs amplitude and frequency analysis on the vibrating structure at the specified frequency.

After that, a regular increment is added to exciter frequency value, and amplitude, the frequency is obtained by using the created functions. From the obtained amplitude values, the one at which the corresponding value of frequency for maximum amplitude is selected and then the code runs to check for amplitude and frequency at +2 increment and -2 increment.

From this second iteration, the frequency at which maximum amplitude was obtained is selected as the resonant frequency. The code shows the dialog box, mentioning that the resonance has been achieved.

The code then continuously operates at the resonant frequency until a drop is detected in the amplitude of the vibrating beam. At this point, the code shows the dialog box that a "drop in amplitude has occurred, therefore resonance frequency is changed". The code then restarts and performs the whole analysis again to calculate the changed resonant frequency of the beam.

\section{RESULTS AND DISCUSSION}

The developed feedback control system code is tested on a cantilever beam with a resonant frequency lower than $15 \mathrm{~Hz}$ due to the limitation of the camera in real-time monitoring. The beam was analyzed using vision system at consecutive alterations of frequency to attain resonance condition. Following are the results which the code calculated and later adjusting the Exciter frequency to vibrate at the resonance frequency.

At first, the exciter starts vibrating at frequency of $6 \mathrm{~Hz}$ and acquires 150 frames. After acquisition the image processing starts and the system calculate frequency, amplitude and error in the image processing system. The error is calculated for the frequency that was set at the start and the frequency that the image processing system calculated. This is done to check the robustness and accuracy of the image processing system. The error was calculated using Eq. 4. The results can be seen in Figure 9.

\footnotetext{
Error $\%=\frac{\mid \text { Actual Frequency }- \text { Frequency measured by MATLAB } \mid}{\text { Actual Frequency }} * 100 \quad$ Eq. (4)
}

After calculating results at $6 \mathrm{~Hz}$, the system then repeats the process at $10 \mathrm{~Hz}$ and $14 \mathrm{~Hz}$. The results can be seen in Figure 10 and 11 .

As can be seen from the figures, the code determines that the maximum amplitude is being obtained at the frequency of 14 $\mathrm{Hz}$. However, it cannot conclude that $14 \mathrm{~Hz}$ is the resonant frequency as it could be \pm 3 of $14 \mathrm{~Hz}$.

Therefore, the code would now check for amplitudes at 12, 13, 15 and $16 \mathrm{~Hz}$ to check whether $14 \mathrm{~Hz}$ was the resonant frequency or one of the adjacent frequencies is resonant frequency. The frequency and amplitude results are visible in Figure 12, 13 and 14.

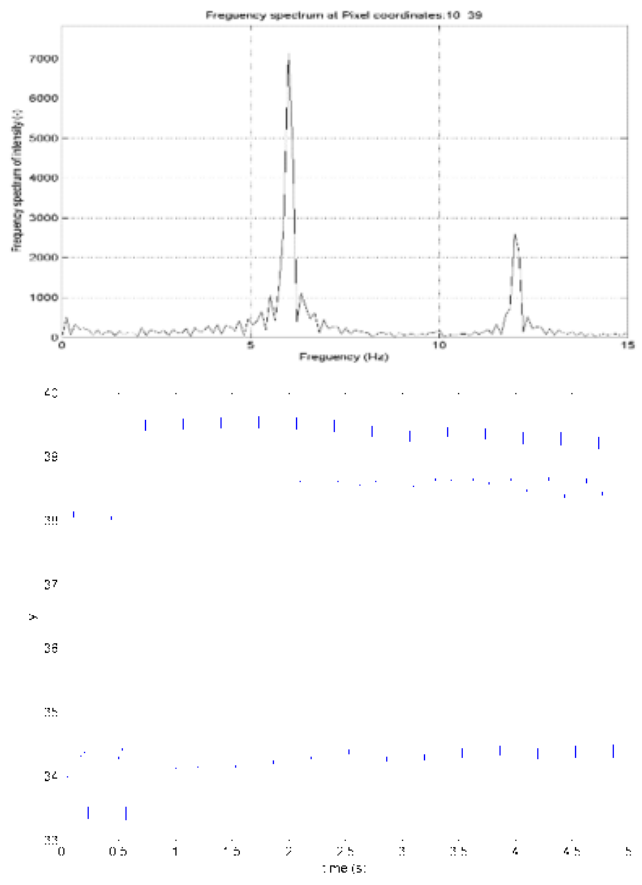

Fig. 9: Frequency and amplitude at $6 \mathrm{~Hz}$

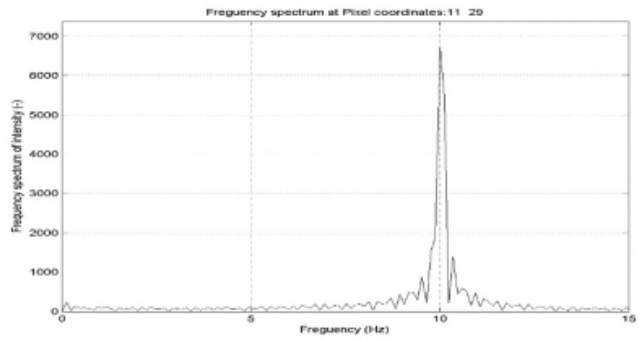




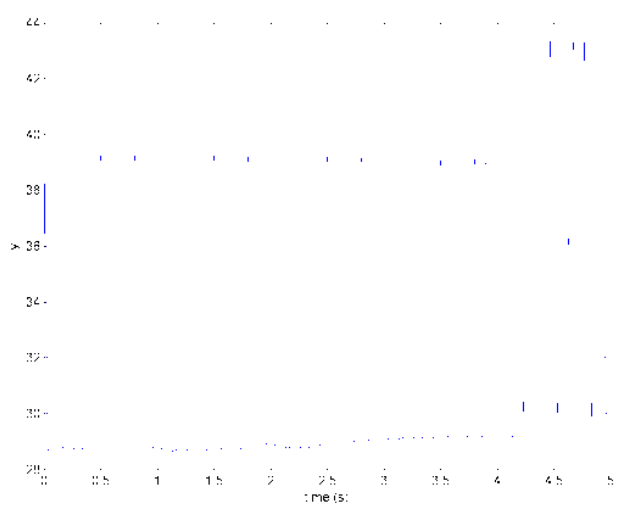

Fig.10: Frequency and amplitude at $10 \mathrm{~Hz}$.
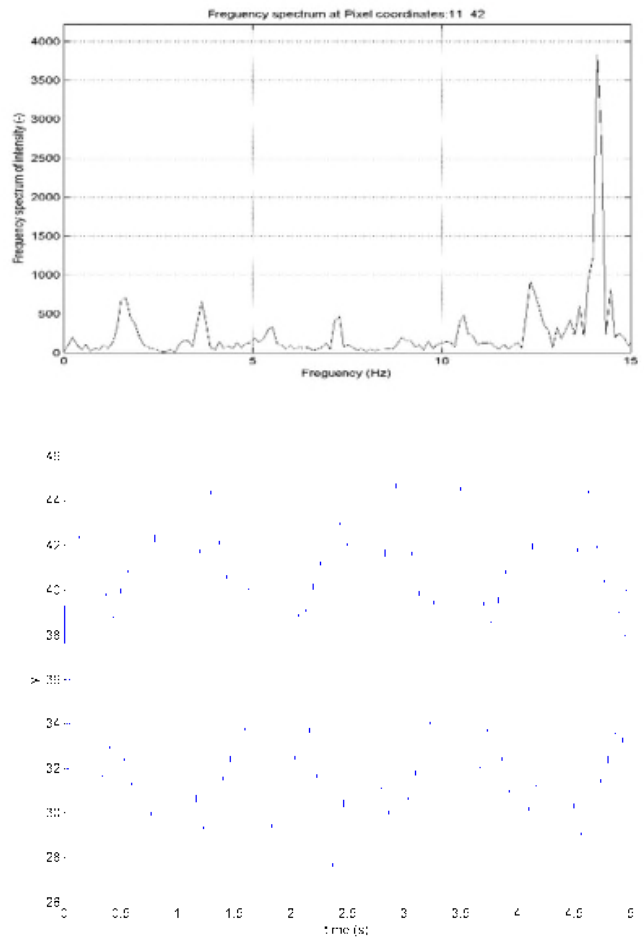

Fig. 11: Frequency and amplitude at $14 \mathrm{~Hz}$

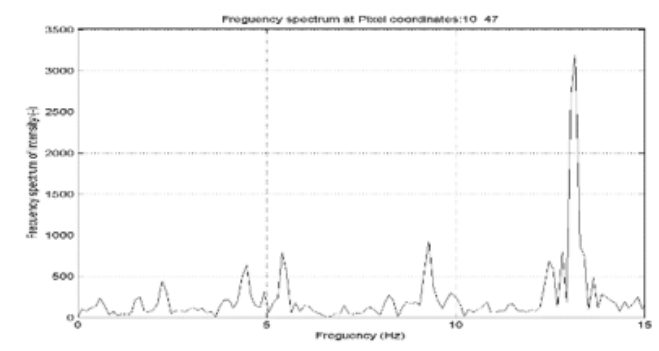

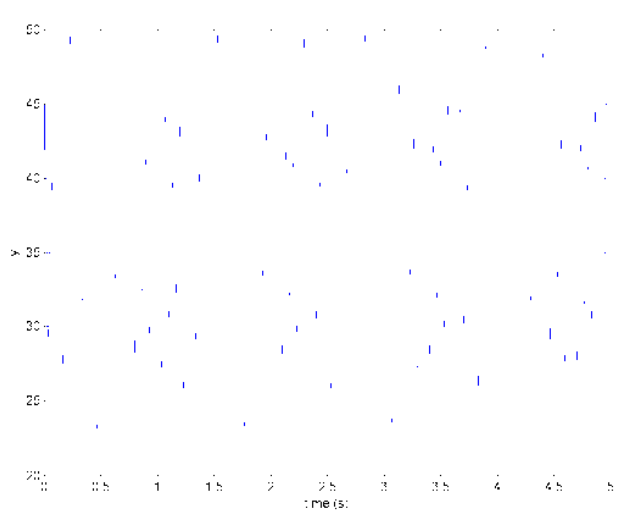

Fig. 12: Frequency and amplitude at $12 \mathrm{~Hz}$
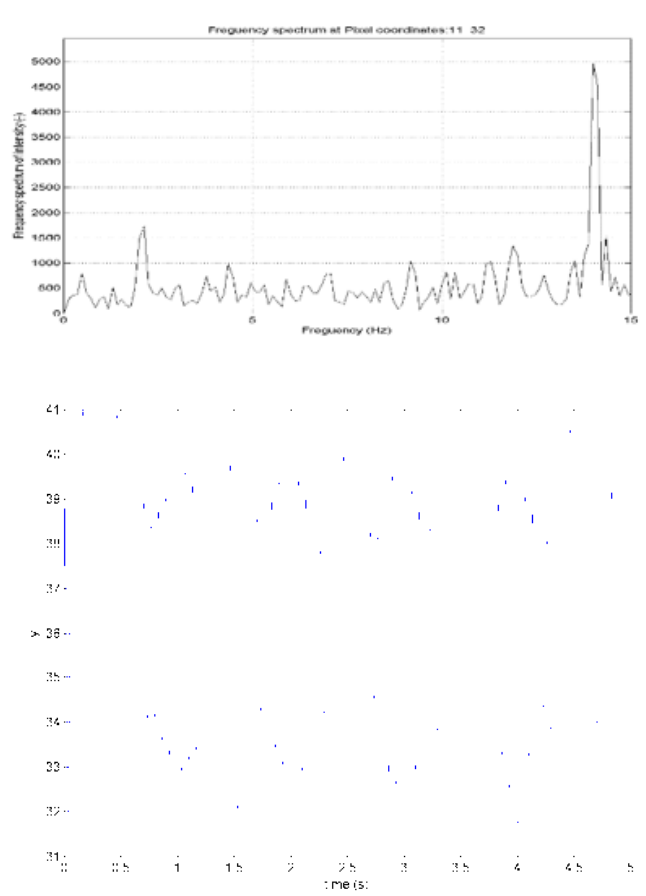

Fig. 13: Frequency and amplitude at $13 \mathrm{~Hz}$

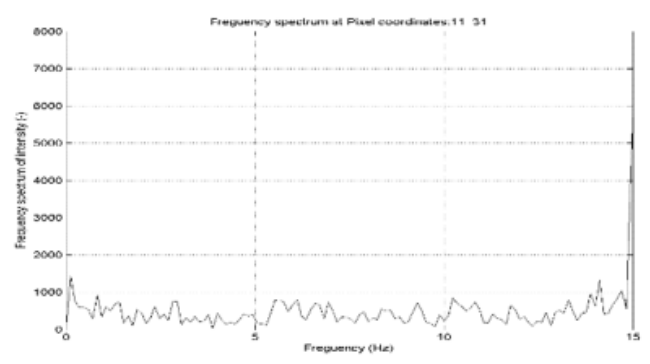




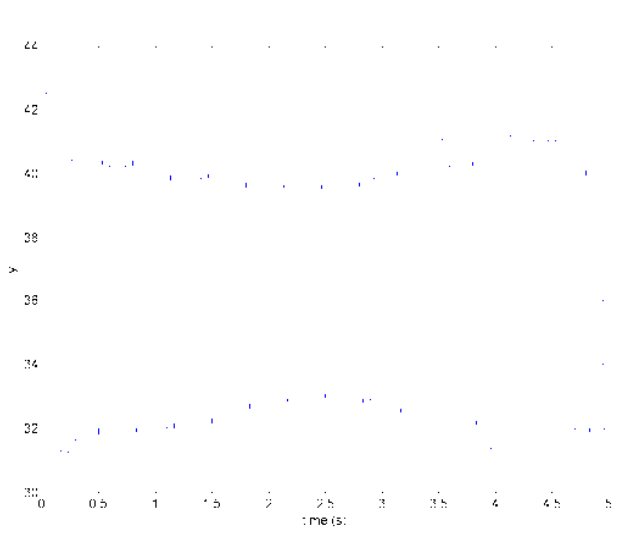

Fig. 14: Frequency and amplitude at $15 \mathrm{~Hz}$

After 2 Iterations, the code concludes that the maximum amplitude is obtained at $12 \mathrm{~Hz}$ and then keeps vibrating at 12 $\mathrm{Hz}$ until a drop in amplitude is detected. Figure 15 shows all the plotted frequency values and shows how resonant frequency is achieved, which is the last point in the graph.

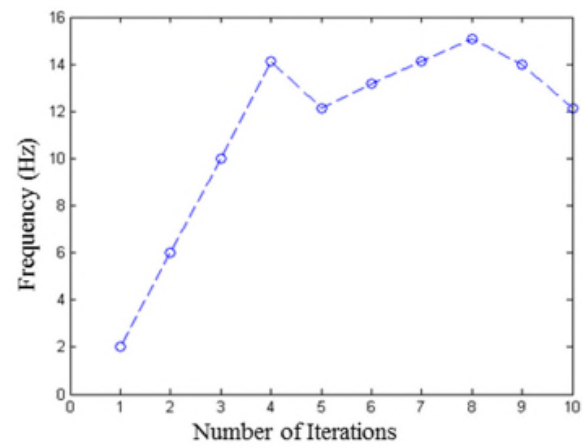

Fig. 15: All calculated frequency values towards resonant frequency at $12 \mathrm{~Hz}$.

The graph clearly depicts how it followed the process to detect resonant frequency, i.e. by adding increments and then when the value drops it would move to and fro to determine the maximum amplitude point.

When a crack is formed or the amplitude of beam changes due to any surrounding changes the code detects a drop in amplitude and gives the message that amplitude drop has been observed and shifts to repeat the entire process again until the frequency is obtained at which maximum amplitude occurs.

The fundamental frequency of the beam calculated using Eq. 1 was $12.4 \mathrm{~Hz}$, whereas the frequency determined by image processing was $12 \mathrm{~Hz}$. It is because the microcontroller is generating a frequency in whole numbers. The microcontroller can be programmed to generate frequency up-to few decimals.

\section{CONCLUSION}

In this paper, a standard procedure followed for performing real-time amplitude and frequency analysis is presented along with experimental results. Apart from our basic theoretical knowledge, fundamental principles of engineering along with basic information of control systems are utilized, the procedure also utilizes the extensive application of MATLAB computer vision toolbox as an interface between image acquisition device and a feedback controller.

Using this toolbox and considering the design consideration of cantilever beam and a feedback controller, a system was proposed which met the deliverables and requirements keeping all the constraints in mind.

The proposed system was tested against different samples of the cantilever beam. It was done to ensure system stability, accuracy and efficiency under different loading conditions.

The key purpose of the system was to evaluate the natural frequencies of the structure using non-contact based techniques so that it could be used to detect natural frequencies of lightweight structures whose frequency cannot be accurately detected using contact based devices.

\section{REFERENCES}

[1] F. M. A. Nogueira, F. S. Barbosa, and L. P. S. Barra, "Evaluation of structural natural frequencies using image processing," Proc. EVACES, 2005.

[2] M. Romaszko, B. Sapiński, and A. Sioma, "Forced vibrations analysis of a cantilever beam using the vision method," J. Theor. Appl. Mech., p. 243, Feb. 2015 .

[3] R. Prislan, "Laser Doppler vibrometry and modal testing." Apr-2008

[4] B. Ferrer, J. Espinosa, A. B. Roig, J. Perez, and D. Mas, "Vibration frequency measurement using a local multithreshold technique," Opt. Express, vol. 21, p. 26198, Nov. 2013.

[5] T. Harčarik, J. Bocko, and K. Masláková, "Frequency Analysis of Acoustic Signal using the Fast Fourier Transformation in MATLAB," Procedia Eng., vol. 48, pp. 199-204, 2012.

[6] X. Shen and J. P.-Y. Maa, "A camera and image processing system for floc size distributions of suspended particles," Mar. Geol., vol. 376, pp. 132 146, Jun. 2016.

[7] J. G. Chen, N. Wadhwa, Y.-J. Cha, F. Durand, W. T. Freeman, and O. Buyukozturk, "Structural Modal Identification Through High Speed Camera Video: Motion Magnification," in Topics in Modal Analysis I, Volume 7, J. D. Clerck, Ed. Springer International Publishing, 2014, pp. 191-197.

[8] N. Ab Azar, A. Babakhani, A. Broumandnia, and K. Sepanloo, “A novel method for detecting and counting overlapping tracks in SSNTD by image processing techniques," Radiat. Meas., vol. 91, pp. 36-43, Aug. 2016.

[9] X. Zhu, S. Ai, D. Fang, B. Liu, and X. Lu, "A novel modeling approach of aluminum foam based on MATLAB image processing," Comput. Mater. Sci., vol. 82, pp. 451-456, Feb. 2014.

[10] M. A. Khan, S. Z. Khan, W. Sohail, H. Khan, M. Sohaib, and S. Nisar, "Mechanical fatigue in aluminium at elevated temperature and remaining life prediction based on natural frequency evolution," Fatigue Fract. Eng. Mater. Struct., 2015 
2019-03-18

Frequency and amplitude measurement of a cantilever beam using image processing: with a feedback system

Khan, Sohaib Z.

IEEE

Khan SZ, Ahmed U, Qazi S, et at., (2019) Frequency and amplitude measurement of a cantilever beam using image processing: with a feedback system. In: IBCAST - International

Bhurban Conference on Applied Science and Technology, 8-12 January 2019, Islamabad, Pakistan https://doi.org/10.1109/IBCAST.2019.8667167

Downloaded from Cranfield Library Services E-Repository 